\title{
On Natural Mobility Models
}

\author{
Vincent Borrel, Marcelo Dias de Amorim, and Serge Fdida \\ LIP6/CNRS - Université Pierre et Marie Curie, \\ 8, rue du Capitaine Scott - 75015 - Paris - France \\ \{borrel, amorim, sf\}@rp.lip6.fr
}

\begin{abstract}
There is an increasing consensus that existing mobility models, such as the well-known random walk or random waypoint models, are insufficient to represent real node mobility. In this paper, we discuss the need for a better characterization of natural mobility. Our contributions rely on recent advances of real-life network analysis and modelling, and in particular on the observation that natural networks behave on a scale-free basis. We devise then a novel mobility modelling approach that focuses on the behavioral aspect of individuals and the interactions between them. This fulfils a gap between individual and group mobility models. Our first results show a strong relevance of the scale-free distribution in mobility modelling, and open further directions in modelling the costs associated to building a network structure in general.
\end{abstract}

\section{Introduction}

The increasing demand for mobile networking has raised a number of complex problems ever addressed by the network research community. Many of these problems do not have all the required elements for a complete solution.

In this context of autonomic communications, mobility management will play a major role. Indeed, given the rapid growth of the radio equipped population, future networks will face serious challenges in terms of node density and complexity of the communication environments. A key functionality to adapting to rapid changes in the environment is to tailor network configuration and routing algorithms according to the spatial characteristics of the real world they rely upon. Considering that a part of future networks are based on mobile terminals, a mobility model matching reality at its best is highly demanded $[1,2]$.

Existing mobility models are either too simplistic or do not represent the real characteristics of user mobility. The current model used to represent mobile scenarios is the Random Waypoint Model, despite its obvious flaws and the lack of similarity it has with real-life situations [3]. We acknowledge that it is difficult to define the real characteristics that a mobility model should capture. We adopt an extrapolative approach by inferring mobility from observations made in reallife networks. Starting from the simple parallel analysis between man-to-group interactions and dynamic principles of real-life network models, we devised a mobility model from the ground up, bridging the gap between individual and

The original version of this chapter was revised: The copyright line was incorrect. This has been corrected. The Erratum to this chapter is available at DOI: 10.1007/978-3-540-32993-0_29

I. Stavrakakis and M. Smirnov (Eds.): WAC 2005, LNCS 3854, pp. 243-253, 2006.

(c) Springer-Verlag Berlin Heidelberg 2006 
group mobility models. The objectives are to bring new enhancements and finegrained population modelling by matching the main observation spanning many real-life domains: scale-free spatial distribution.

Such a model could be useful in many aspects. Being a behavioral model, it may give us clues on some statistics of the behaviors it implements. This may serve, for example, as a feedback to sociologists to validate hypothesis on dense and large populations, which might be difficult to measure in practice. In the context of autonomic communications, it would serve as a basis for innovative heuristics for routing, connectivity establishment, self-healing, and security. With this work, we also intend to strengthen the links among complementary disciplines. We are interested, in particular, in how biological or sociological observations could be integrated in our framework.

The remainder of this paper is organized as follows. In Section 2, we survey existing mobility models. In Section 3, we focus on the explanation of the most distinctive characteristics of real-life networks, and quickly overview where such characteristics have been observed. We summarize, in Section 4, the considerations that led us to reconsider mobility models, and describe the objectives, characteristics, and first results concerning our approach. Finally, Section 5 concludes this paper.

\section{An Overview of Existing Mobility Models}

A number of mobility models have been proposed in the literature [1]. Generally, two types of mobility have been addressed so far: individual mobility and group mobility. We define them in the following and present the most important approaches proposed in the literature.

\subsection{Individual Mobility Models}

Individual mobility deals with the movement at the node level, where each node is considered independently from the others. We present in the following the most important models proposed so far.

The Random Walk mobility model. First proposed by Albert Einstein in 1926 to characterize Brownian motion, is also called "Drunkard's Walk", and is the de facto mobility model used for mobile network analysis. In this model, a node travels by changing its direction and speed at random, at regular time or distance intervals. However, since its behavior is independent of past motion (memoryless), it generates very unrealistic displacements.

The Random Waypoint mobility model. In this model, nodes travel between randomly chosen locations. The speed of displacement and pause periods are also randomly determined. This model is also widely used in mobile network simulations; however, since the performance is obtained in a bounded space, the density of nodes at the center of the simulation area tends to grow indefinitely.

The Random Direction mobility model. This model has been conceived to overcome this drawback of the Random Waypoint model. Here, a node chooses 
a random direction and follows it until it reaches a border, pauses there for a random duration, then restarts by choosing its next direction. The problem is that nodes tend to stay at the borders of the simulation area, which is likely to generate network partitions and big hop counts in simulated networks.

The Boundless Simulation Area mobility model. It focuses on a boundless space, by wrapping a rectangular zone around its opposite borders, in a toroidal manner. Each node has a direction and a speed, respectively updated at random following a maximal angular change speed and a maximal acceleration. This model has an even spatial repartition, and accounts for a quite realistic user movement. However, it does not account for pauses, and the boundless situation is not considered to be the most representative one in simulations of mobile scenarios.

The Gauss-Markov mobility model. This model considers that nodes have an initial speed and direction, which they update at each time step, taking into account the previous speed/direction $\left(s_{n-1} / v_{n-1}\right)$, the mean speed/direction $(\bar{s} / \bar{v})$, and a random value $\left(s_{x_{n-1}} / v_{x_{n-1}}\right)$ having a Gaussian distribution. These parameters are considered with different weights, according to a randomness parameter $\alpha$. The current speed and direction are then given by:

$$
\begin{aligned}
& s_{n}=\alpha s_{n-1}+(1-\alpha) \bar{s}+\sqrt{1-\alpha^{2}} s_{x_{n-1}}, \\
& d_{n}=\alpha d_{n-1}+(1-\alpha) \bar{d}+\sqrt{1-\alpha^{2}} d_{x_{n-1}} .
\end{aligned}
$$

The City Section mobility model. This model uses a street network map, upon which nodes go from random place to random place choosing their shortest time path, with possible speed limitations and minimal distance between nodes, and pause upon arrival before restarting. It is very specific, being designed to render cars or pedestrians in constrained maps.

\subsection{Group Mobility Models}

In a group mobility model, the mobility of a node is computed relatively to the mobility of a reference point in the subset of nodes (group) it belongs to. A number of such models have been proposed in the literature, and some of them are described in the following.

The Reference Point group mobility model. It is the most generic group mobility model. It implements groups of nodes which follow more or less loosely a reference point whose motion can be dictated by various ways, such as using one of the previously discussed individual mobility models. Its specialization gives birth to mobility models presented in the following.

The Exponential Correlated mobility model. This model uses a motion function to compute the next movement vector $\vec{b}(t)$, in the complex space, given by: 


$$
b(t+1)=b(t) e^{-\frac{1}{\tau}}+\left(\sigma \sqrt{1-e^{-\frac{2}{\tau}}}\right) r,
$$

where $r$ is a random Gaussian variable of variance $\sigma$, and $\tau$ accounts for the rate of change (the smaller $\tau$, the quicker the change). The main problem here is that it is difficult to set appropriate parameters to obtain a particular effect.

The Nomadic Community mobility model. In this model, groups move from point to point, inside which every member wanders in a Brownian motion locally. It corresponds in fact to a combination of the Random Waypoint that uses locally, in a smaller scale, a Random Walk.

The Pursue mobility model. This model defines a particular node which is the target, while the other ones are its prosecutors. The target follows its own mobility model while the other nodes have a motion accelerating toward it, plus a random vector.

\section{Aspects of Real-Life Networks}

Many recent studies have found, in various areas of real-life ranging from biology to computer networks, via sociology, scientific citation, literature, movie acting, ecosystems or economics, some fascinating common features of the networks, or graphs, modelling the many relationship that pervade them. These common features, not present in the traditional Erdös-Rényi Random graph model, lie on two major aspects: the scale-free property and the high clustering coefficient.

The scale-free property relates to a power-law distribution of the degrees of nodes in the network. This distribution is different from the usual Poisson distribution, also called exponential, defining node degrees in the random network model.

The power law distribution means that the probability of having a node of degree $k$ is

$$
P[k] \propto k^{-\lambda},
$$

where $\lambda$, the exponent, can be seen in a log-log graph as the pent of the linear fit of the distribution. Fig. 1 shows an example of the resulting node distribution for both the random and scale-free cases.

The clustering coefficient defines the propensity of nodes to be gathered in small groups that are highly interconnected. Its mathematical formula is derived from the "fraction of transitive triples", defined by Wasserman and Faust in [4]. At the node level, it is the effective number $E_{i}$ of links relating the node's $k_{i}$ direct neighbors over the total possible number of links between them:

$$
C_{i}=\frac{2 E_{i}}{k_{i}\left(k_{i}-1\right)} .
$$

Averaged over all its nodes, $C_{i}$ becomes the clustering coefficient of a graph. In random graphs, as each edge exists with a probability $p$, the clustering coefficient is $C=p$. In many real-life networks, it has been found to be several orders of magnitude higher than in random graphs. 

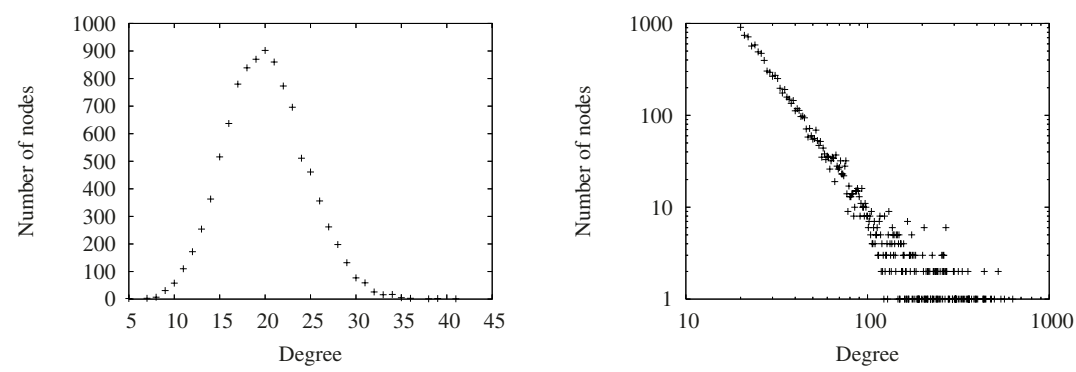

Fig. 1. A comparison of Random graphs versus Scale-Free graphs. On the left, the node degree distribution of an Erdös-Rényi random graph with 10000 nodes and a link probability of 0.02 , in a linear scale. On the right, the node degree distribution of an Albert-Barabási scale-free graph grown to 10000 nodes with preferential attachment in a log-log scale. Both have the same number of nodes and links, but their structures are significantly different.

Various observations have been made of these two characteristics in many domains, as presented in the following.

\subsection{Biology}

Jeong et al. [5] studied the metabolism of 43 organisms in networks where the nodes are substrates (ATP, ADP, $\mathrm{H}_{2} \mathrm{O}, \ldots$ ) and edges account for the predominantly directed chemical reactions in which these substrates participate. The distribution of incoming and outgoing edges are both in power-law with respective degrees of 2.4 and 2. Wagner and Fell [6] concentrated on the metabolism of Escherichia coli bacterium. Here in addition to a power-law distribution, the undirected version of the substrate graph has a small average path length and a high clustering coefficient (around ten times that of an equivalent random graph).

Another important network is the one of interactions between proteins. Here the nodes are proteins, and the edges represent the fact that they bind together. Jeong, Mason et al. [7] studied such a network in the yeast (S. cerev.) and found a power-law with an exponent of 2.4.

Scala, Amaral and Barthélémy [8] studied the networks formed by the conformation of a two-dimensional lattice polymer. The clustering coefficient is much larger than the one of an equivalent random graph. However, here, the degree distribution is consistent with a Gaussian.

The network of gene expression (here a node represents the expression of a gene, and the impact that the expression of a gene has on the expression of another gene is an edge) also exhibits power-law distributions.

\subsection{Computer Networks}

In the Internet, partial graphs, both at the router and domain levels, have been shown to have the scale-free property, with a degree distribution of the nodes in 
power law, with an exponent between 2 and $3[9,10]$. At the domain level, the clustering has also been shown to be two to three orders of magnitude higher than in random graphs of the same size.

Similar results have been observed in the context of the Web (hyperlinks). Although being a directed graph, many studies found both its in-degree and outdegree distributions to be following a power-law of the same exponent range, also between 2 and $3[11,12]$. Furthermore, the clustering coefficient was also found [13] to be between 2 and 3 orders of magnitude higher than the ones of a similar random graph.

\subsection{Sociology}

This domain is one of the most prolific, and also the oldest. It relates directly to humans, and network structures depend on their behaviors.

The Internet Movie Database [14] contains all movies and their casts since the 1890s. Here the nodes are actors and two actors having participated in a movie have an edge between them. This graph can also be seen as a bipartite graph, consisting of a set of actors, a set of movies, and a set of participations, linking an actor to a movie. Watts and Strogatz, in their study of 1998 [15] found its clustering coefficient to be 0.79 , around 3000 times more than the one of the equivalent random graph, 0.00027 .

Citation networks in scientific publications are made of nodes which represent publications, and edges that go from a publication to the other one it cites. The first study of scale-free properties in citation networks of scientific publications were conducted in 1965 by Derek de Solla Price. He noted that these networks have power-law distributions, and subsequently built a model, close to the one of Albert and Barabási, but directed and adapted to publications networks that are directed. A more recent study by Redner [16] found that the in-degree of these networks follow a power-law distribution, with an exponent of 3 .

In phone call networks, where nodes are phone numbers and edges telephonic conversations between them are a kind of social network. Abello et al. [17] and $\mathrm{Lu}[18]$ studied the graphs of long distance telephone calls made during a day and found that the distributions of incoming and outgoing calls follows a power-law with an exponent of 2.1 .

\subsection{The Albert-Barabási Model}

As shown by Albert and Barabási in their eponym model [11,19], scale-free distributions can be obtained mimicking the dynamics of groups of elements following two simple rules: growth and preferential attachment. Albert and Barabási start from an initial random network, and progressively add new nodes. This is the growth principle. In the preferential attachment principle, the probability that a new node be connected to an existing node $i$ is proportional to $i$ 's connectivity degree:

$$
\Pi\left(k_{i}\right)=\frac{k_{i}}{\sum_{j} k_{j}}
$$


This process gives rises to graphs with scale-free degree distributions, as illustrated in figure 1.

They also showed the generated graphs to be more resilient to random failures [20].

\section{A Novel Approach for Mobility Modelling}

In the models presented in Section 2, complex interactions are faintly represented, if not at all. For example, in group mobility models, groups are fixed and cannot evolve in time. Zhou et al. [21] address this issue by creating the Group and Swarm Mobility model, where groups follow virtual tracks and swarm at intersections. However, to be able to regroup at intersections, two subgroups must arrive at an intersection at the same time. This constraint leads to a plethora of small groups, and large groups cannot be well represented.

Furthermore, none of these models minds the spatial density dynamics of populations. In real-world situations, groups forms and dislocate, crowds evolve, people mimic others, the total population grows or diminishes. Another point is that the notion of group is too rigid for many situations. Nodes must belong to a precise group, and only one, or be independent. Group don't evolve, and moreover, the behavior space is discretized in few classes, if more than one, which doesn't allow for fine behavior materialization. We would like to fine-tune the tendency to behave more or less following one or more groups.

\subsection{Why Scale-Free Characteristics in Mobility?}

It is now a known fact that mobility strongly influences the results of ad-hoc protocols simulations. As there currently exist no precise large scale traces of users mobility, one is forced to resort to the mobility models for such work.

A mobility model must be as close as possible to the reality, and thinking about matching reality we must strongly consider its prevalent characteristics. As we have seen earlier, one of these prevalent characteristics has been extensively reported in many different areas. It is the scale-free distribution of graphs representing real-life situations.

Although this reason is by far not sufficient to justify by itself a Scale-Free behavior in human mobility, several other aspects clearly point to its usefulness.

In the first place, the preferential attachment behavior in human crowds is a known fact in sociology. From the 'rich get richer' comportment, at the base of the Albert-Barabàsi model itself, to the positive feedback of crowd sizes on their growth, passing by the propensity of people to admit the most common idea as the most valid. If then, as in many other aspects, preferential attachment in human motion decision behaviors can be found, the question of whether it drives to scale-free spatial density distributions becomes prominent.

This supposition becomes especially relevant in the light of recent studies by Chaintreau et al. [22], which observe scale-free inter-contact distributions in different crowds of humans equipped with Bluetooth devices. In fact, scale-free spatial distributions might greatly account in these surprising observations. 
This is very important since mobile ad-hoc networks structures are highly dependant on the connectivity of the nodes, itself depending on the spatial density of them. If this density is scale-free, the network structure inherited will be far different from a random network, and knowing this property can greatly help design routing protocols, prior to help better test them.

The lacunas we have seen in existing mobility models led us to consider a new category of mobility model, where a collective behavior arises. Such a model must have, however, a finer-grained definition of the traditional concept of group, which is too rigid to efficiently represent the evolution of real populations. This new type of mobility is called gathering mobility, where nodes meet in space and evolve almost independently one from another (with some level of interaction).

We want to model displacement of crowds in free spaces, with centers of interests. Such a model finds its application in many scenarios, as for example in an exhibition hall, where different booths exist. This can also represent a school courtyard, where children go from occupation to occupation, from group to group. This can also be seen as a market or shopping center, where people aggregate around stores and stands. More generally, this can be seen as the way people go: from an interesting place to another interesting place. This model would have people tending to judge the interest of a place in function of the interest other people have in this place, following trends and mimicking others.

\subsection{Characterization of the Model}

We consider the network as a constrained rectangular space of configurable dimensions, where two different types of objects coexist: individuals and attractors. ${ }^{1}$

Attractors are landmarks toward which nodes move; they appear for a certain period of time, do not move, then disappear. They model centers of interest for individuals (e.g., stands in a show). Each attractor is associated with a force, which influences its propensity to gather individuals.

Individuals are the main focus of our work. They behave in cycles. A cycle consists of a displacement, a pause period, and a decision of leaving or not the current position. Displacements are characterized by the origin and a target attractor. We will show that in the choice of the target lies the main characteristic of the model.

The preferential attachment principle is implemented in the attractor decision process as follows. The probability $\Pi\left(a_{i}\right)$ that an individual $z_{k}$ chooses an

\footnotetext{
${ }^{1}$ The term attractors used here bears similarities with another term, Attraction points, defined by Jardosh et al. in [23]. They both model centers of interest for nodes displacements. However, their function and the mobility they generate are different in essence. In the Jardosh paper, nodes move from point to point along enhanced Voronoi graph. During some time intervals, a random selected Voronoi point becomes and attraction point, the destination of a certain percentage of all the nodes. In our proposal, Nodes or Individuals move freely in space, and all destination points are Attractors. Our mechanism implements positive feedback, in the form of preferential attachment to select the desired attractor among others, weighted by inverse of distance.
} 
attractor $a_{i}$ among all possible ones is proportional to the portion of the total attractiveness it carries: $\Pi\left(a_{i}\right)=\frac{\mathcal{A}_{a_{i}, z_{k}}}{\sum_{j} \mathcal{A}_{a_{j}, z_{k}}}$. The attractiveness of an attractor is relative to an individual, and is proportional to its popularity (number of other individuals who chose it), and inversely proportional to the distance separating it from the considered individual:

$$
\mathcal{A}_{\left(z_{i}, a_{l}\right)}=\frac{\left(1+\sum_{z_{j} \in \mathbf{Z}, z_{j} \neq z_{i}} B\left(z_{j}, a_{l}\right)\right)}{\sqrt{\left(X_{a_{l}}-X_{z_{i}}\right)^{2}+\left(Y_{a_{l}}-Y_{z_{i}}\right)^{2}}} .
$$

where $B\left(z_{j}, a_{l}\right)$ is a Bernoulli variable, with $B=1$ if the individual $z_{j}$ is going toward or staying at attractor $a_{l}$ and 0 otherwise, and $\mathrm{X}$ and $\mathrm{Y}$ are the coordinates of a node (individual or attractor). Observe that Eq. 7 includes the effect of other nodes' decision on $z_{i}$ to represent the collective behavior of individuals.
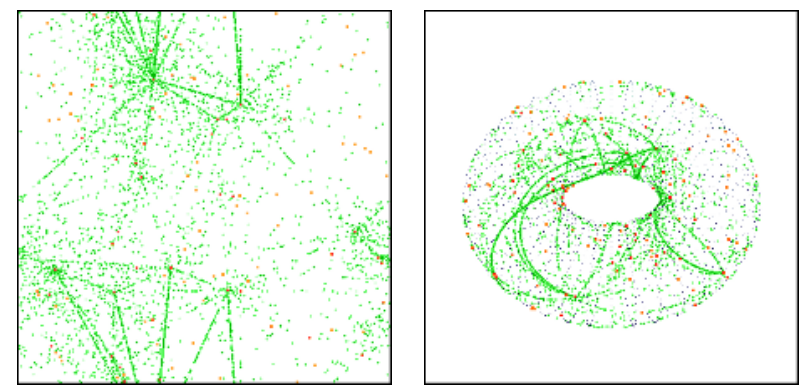

Fig. 2. A view of the simulated mobility showing the spatial distribution of nodes in both planar and toroidal representations. One can clearly see paths forming, and a much diversified spatial distribution of individuals.

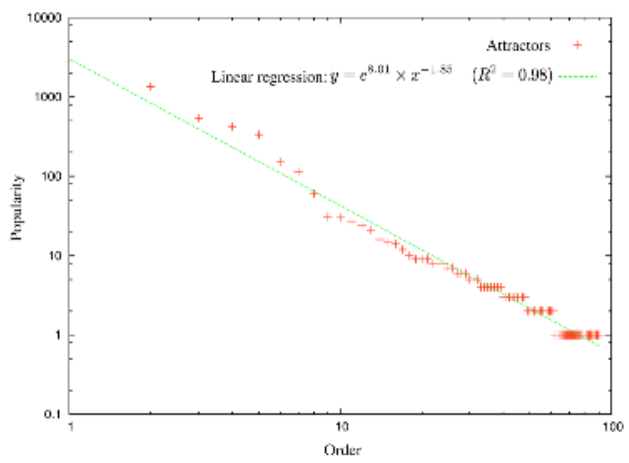

Fig. 3. First results of our model, in situation of population growth 


\subsection{Experiments}

We have built a simulator to test the premises of our mobility model (see screenshots shown in Fig. 2). The scale-free nature of the Albert-Barabási model was observed in situation of population growth. Thus, we place our model in this same context, where attractors and individuals arrive and never leave.

In a simulation run of 10,000 individuals, we obtain the distribution shown in figure 3. A linear fit in log-log scale gave us a power-law distribution, with an exponent of -1.85 and a confidence of 0.98 . These results exhibit the scale-free characteristic of the attractor population.

A corollary result is that, given an equiprobable spatial distribution of nodes, and in situation of population growth, the preferential attachment, even weighted by the inverse of distance, gives scale-free results.

\section{Conclusion}

Following observations in nature of a scale-free distribution, we devised a mobility model using preferential attachment, aimed at fulfilling lacunas seen in the domain of mobility modelling. This mobility model belongs to a new kind of mobility paradigm, we call gathering mobility. In this model, individuals evolve independently and do not explicitly belong to groups, although they exhibit strong collective behavior and are influenced by others. They gather around centers of interest of varying popularity levels.

Our first tests show that, in situation of growth, such as defined by recent scale-free models, our mobility model leads to scale-free spatial density, where preferential attachment is weighted by inverse of distance.

Further works will focus on the characterization of the parameter space of our model. We will investigate in particular other types of population dynamics: not only growth, but also steady and renewing populations, as well as decreasing ones. In these situations it is important to verify if the scale-free distribution of attractors is maintained.

\section{References}

1. T. Camp, J. Boleng, and V. Davies, "A survey of mobility models for ad hoc network research," Wireless Communications and Mobile Computing (WCMC), vol. 2, no. 5, no. 5, pp. 483-502, 2002.

2. M. E. J. Newman, "The structure and function of complex networks," SIAM Review, vol. 45, no. 2, no. 2, pp. 167-256, 2003.

3. J. Yoon, M. Liu, and B. Noble, "Random waypoint considered harmful," 2003.

4. S. Wasserman and K. Faust, "Social network analysis : Methods and applications," Cambridge university, 1994.

5. H. Jeong, B. Tombor, R. Albert, Z. N. Oltvai, and A.-L. Barabási, "The large-scale organization of metabolic networks," Nature, vol. 407, pp. 651-654, 2000.

6. D. A. Fell and A. Wagner, "The small world of metabolism," Nature Biotechnology, vol. 18, pp. 1121-1122, 2000. 
7. H. Jeong, S. Mason, A.-L. Barabási, and Z. N. Oltvai, "Lethality and centrality in protein networks," Nature, vol. 411, pp. 651-654, 2001.

8. A. Scala, L. A. N. Amaral, and M. Barthélémy, "Small-world networks and the conformation space of a lattice polymer chain," Europhys. Letters, vol. 55, pp. 594600, 2001.

9. M. Faloutsos, P. Faloutsos, and C. Faloutsos, "On power-law relationships of the internet topology," pp. 251-262, 1999.

10. R. Govindan and H. Tangmunarunkit, "Heuristics for internet map discovery," Proceedings of IEEE Infocom 2000, vol. 3, p. 1371, 2000.

11. R. Albert, H.jeong, and A.-L. Barabási, "Diameter of the world-wide web," Nature, no. 401, pp. 130-131, Sept. 1999.

12. R. Kumar, P. Raghavan, S. Rajagopalan, D. Sivakumar, A. S. Tomkins, and E. Upfal, "Stochastic models for the web graph," in Proceedings of the 42nd Annual IEEE Symposium on the Foundations of Computer Science, (NY), pp. 57-65, IEEE, 2000.

13. B. A. Huberman and L. A. Adamic, "Growth dynamics of the world-wide web," Nature, no. 401, p. 131, Sept. 1999.

14. "Internet movie database." http://www.imdb.com.

15. D. J. Watts and S. H. Strogatz, "Collective dynamics of 'small worlds' networks," Nature, vol. 393, pp. 440-442, 1998.

16. S. Redner, "How popular is your paper ? an empirical study of the citation distribution," Eur. Phys. J. B, vol. 4, pp. 131-134, 1998.

17. W. Aiello, F. Chung, and L. Lu, "A random graph model for massive graphs," in Proceedings of the 32nd Annual ACM Symposium on Theory of Computing (A. of computing machinery, ed.), (New York), pp. 171-180, 2000.

18. L. Lu, "The diameter of random massive graphs," in 12th Annual Symposium on Discrete Algorithms (SODA), ACM-SIAM.

19. R. Albert and A.-L. Barabási, "Statistical mechanics of random networks," Review of Modern Physics, vol. 74, pp. 47-97, 2002.

20. R. Albert, H. Jeong, and A.-L. Barabási, "Attack and error tolerance of complex networks," Nature, vol. 406, pp. 378-382, 2000.

21. B. Zhou, K. Xu, and M. Gerla, "Group and swarm mobility models for ad hoc network scenarios using virtual tracks," (Monterey, California, USA), 2004.

22. A. Chaintreau, P. Hui, J. Crowcroft, C. Diot, R. Gass, and J. Scott, "Pocket switched networks: Real-world mobility and its consequences for opportunistic forwarding," tech. rep., Cambridge, UK, Feb. 2005.

23. A. P. Jardosh, E. Belding-Royer, K. C. Almeroth, and S. Suri, "Real-world environment models for mobile network evaluation," Mar. 2005. 